\title{
Drawing Directed Acyclic Graphs: An Experimental Study*
}

\author{
Giuseppe Di Battista ${ }^{1}$, Ashim Garg ${ }^{2}$, Giuseppe Liotta ${ }^{2}$, Armando Parise ${ }^{3}$, \\ Roberto Tamassia ${ }^{2}$, Emanuele Tassinari ${ }^{3}$, Francesco Vargiu ${ }^{4}$, Luca Vismara ${ }^{2}$ \\ 1 Dipartimento di Discipline Scientifiche, Sezione Informatica \\ Università degli Studi di Roma Tre, Italy \\ dibattistaciasi.rm.cnr. it \\ 2 Center for Geometric Computing, Department of Computer Science \\ Brown University, USA \\ $\{$ ag, gl, rt, lv $\}$ ecs. brown. edu \\ 3 Dipartimento di Informatica e Sistemistica \\ Università degli Studi di Roma "La Sapienza", Italy \\ \{parise,tassinar\}edis . uniroma1.it \\ 4 Autorità per l'Informatica nella Pubblica Amministrazione \\ Italy \\ vargiveaipa.it
}

\begin{abstract}
In this paper we consider the class of directed acyclic graphs (DAGs), and present the results of an experimental study on four drawing algorithms specifically developed for DAGs. Our study is conducted on two large test suites of DAGs and yields more than 30 charts comparing the performance of the drawing algorithms with respect to several quality measures, including area, crossings, bends, and aspect ratio. The algorithms exhibit various trade-offs with respect to the quality measures, and none of them clearly outperforms the others.
\end{abstract}

\section{Introduction}

Over a decade of research in the graph drawing area, motivated by applications to information visualization, has produced a wealth of drawing algorithms. Assessing the practical performance of some of these algorithms has been recently the goal of experimental comparative studies.

In [18] the performance of four planar straight-line drawing algorithms is compared. The standard deviations in angle size, edge length, and face area are used to compare the quality of the planar straight-line drawings produced. Since the experiments are limited to randomly generated maximal planar graphs, this

* Research supported in part by the National Science Foundation under grant CCR9423847, by the U.S. Army Research Office under grant DAAH04-96-1-0013, by ESPRIT LTR of the European Community under Project no. 20244 (ALCOM-IT), by the NATO-CNR Advanced Fellowships Programme, and by a gift from Tom Sawyer Software. 
work gives only partial insight on the performance of the algorithms on general planar graphs.

Himsolt [17] presents a comparative study of twelve graph drawing algorithms. The algorithms selected are based on various approaches (e.g., forcedirected, layering, and planarization) and use a variety of graphic standards (e.g., orthogonal, straight-line, polyline). Only three algorithms draw general graphs, while the others are specialized for trees, planar graphs, Petri nets, and graph grammars. The experiments are conducted with the graph drawing system GraphEd [17]. Many examples of drawings constructed by the algorithms are shown, and various objective and subjective evaluations on the aesthetic quality of the drawings produced are given. However, statistics are provided only on the edge length, and few details on the experimental setting are provided. The charts on the edge length have marked oscillations, due to the small size of the test suite (about 100 graphs). This work provides an excellent overview and comparison of the main features of some popular drawing algorithms. However, it does not give detailed statistical results on their performance.

Di Battista et al. $[7,8]$ present an extensive experimental study comparing four general-purpose graph drawing algorithms. The four algorithms take as input general undirected graphs and construct orthogonal grid drawings. The test graphs are generated from a core set of 112 graphs used in "real-life" software engineering and database applications. The experiments provide a detailed quantitative evaluation of the performance of the four algorithms, and show that they exhibit trade-offs between "aesthetic" properties (e.g., crossings, bends, edge length) and running time. The observed practical behavior of the algorithms is consistent with their theoretical properties.

Brandenburg, Himsolt, and Rohrer [2] compare five "force-directed" algorithms for constructing straight-line drawings of general undirected graphs. The algorithms are tested on a a wide collection of examples and with different settings of the force parameters. The quality measures evaluated are crossings, edge length, vertex distribution, and running time. They also identify trade-offs between the running time and the aesthetic quality of the drawings produced.

Jünger and Mutzel [19] investigate crossing minimization strategies for straight-line drawings of 2-layer graphs, and compare the performance of eight popular heuristics for this problem.

In this paper we consider the important class of directed acyclic graphs (DAGs), and compare the performance of four drawing algorithms specifically developed for them. DAGs are commonly used to model hierarchical structures such as PERT diagrams in project planning, class hierarchies in software engineering, and is-a relationships in knowledge representation systems.

The contributions of this work can be summarized as follows:

- We have developed a general experimental setting for comparing the practical performance of graph drawing algorithms for DAGs. Our setting consists of $(i)$ two large test suites of DAGs, one obtained from the collection of directed graphs submitted to the e-mail graph drawing service at Bell Labs [21], and the other randomly generated by a program that simulates a 
PERT project planner; (ii) a set of quality measures for drawings of DAGs derived from [7].

- Within our experimental setting, we have performed a comparative study of four popular drawing algorithms for DAGs: two of them are based on the layering paradigm $[20,23]$, while the other two are based on the grid paradigm $[10,11]$.

- Our comparison highlights how more than ten years of research in this field have produced a complex landscape. Namely, the four algorithms exhibit various trade-offs with respect to the quality measures, and none of them clearly outperforms the others. The sometimes surprising findings of our investigations include:

- Some algorithms construct very compact drawings at the expense of a relaxed resolution rule that does not consider crossing-crossing and vertex-crossing distances. Other algorithms produce drawings that distribute vertices and crossings with great regularity at the expenses of a larger area requirement.

- Concerning bends, an algorithm with good theoretical worst-case bounds performs in practice worse than algorithms for which no theoretical bounds are available.

- Concerning crossings, grid-based algorithms tend to perform worse than layering-based algorithms, where part of the geometry of the drawing is decided at the very first step.

- The performance of a drawing algorithm on planar DAGs is not a good predictor of the performance of the same algorithm on nonplanar DAGs

- Algorithms with a topological foundation tend to distribute the bends and the lengths of the edges more evenly.

- Our analysis of the performance of the four algorithms has motivated us to develop a new hybrid strategy for drawing DAGs, which uses a layering-based method to perform the initial planarization and a grid-based method to compute the final drawing. The new strategy performs quite well in practice.

The rest of the paper is organized as follows. The four algorithms being compared are described in Section 2. Details on the experimental setting are given in Section 3. In Section 4, we summarize our experimental results in 14 charts, and perform a comparative analysis of the performance of the four algorithms. The new hybrid strategy is described and its performance is discussed in Section 5. Open problems are addressed in Section 6. Due to space limitations we could not include all the charts, which appear in a longer version of this paper [6].

\section{The Drawing Algorithms Under Evaluation}

We have tested four different algorithms for producing drawing of DAGs. Our main graph drawing tool has been $G D W[3]$, a system for prototyping and testing graph drawing algorithms.

The four algorithms can be classified into two categories on the basis of their approach to constructing drawings. 
Layering-Based: These algorithms construct layered drawings, i.e., drawings where the vertices and edge-bends are placed at integer coordinates on a set of horizontal layers, and each edge is drawn as a curve monotonically increasing in the $y$-direction. Note that in such drawings, even though vertices and edge bends are placed at integer coordinates, the edge crossings can be arbitrarily close to each other or to the vertices and edge bends. These algorithms accept as input directed graphs without any particular restriction (the input directed graph can be planar or not, acyclic or cyclic). For constructing drawings, they generally follow the methodology of Sugiyama et al. [23], which consists of the following three steps:

Step 1 Assign vertices to layers heuristically optimizing some criteria, such as the total edge length.

Step 2 Reduce the crossings among edges by permuting the order of vertices on each layer.

Step 3 Reduce the number of bends by readjusting the position of vertices on each layer.

Because of their generality and conceptual simplicity, these algorithms are very popular among the designers of practical graph drawing systems. Several layering-based algorithms have been designed [5]. The above steps have also been investigated separately, and various heuristics have been proposed for each of them.

In this paper, we have evaluated and compared the performance of two layering based algorithms: Dot and Layers.

Dot is a highly optimized algorithm, developed by Koutsofios and North [20] as a successor to $\operatorname{Dag}[13,14]$. Dot first constructs a polyline layered drawing of the input directed graph and then, as a final step, converts the polygonal chains representing the edges into smooth curves using splines. An implementation of $D o t$ is available at $f \mathrm{tp}: / / \mathrm{ftp}$. research. att.com/dist/drawdag/, and this is the implementation we used. However, since all other algorithms considered in this study represent edges as polygonal chains, we decided to analyze the polyline drawing produced by $D o t$ and not the final drawing with curved lines.

Layers is the original algorithm by Sugiyama, Tagawa and Toda [23]. For our study we have used the implementation of Layers available in GDW [3].

Grid-Based: These algorithms accept, as input, a planar st-graph, i.e., a planar DAG with exactly one source and one sink, and construct an upward grid drawing of it. In an upward grid drawing the vertices, the edge-bends and the edge-crossings are all placed at integer coordinates, and each edge is drawn as a curve monotonically increasing in the $y$-direction. Although the requirement of having just one source and one sink may appear too restrictive, such directed graphs occur in several practical applications, such as activity planning (where they are called PERT graphs), network flows, etc. These algorithms are also called numbering-based algorithms because they typically construct a numbering of the vertices and faces of the planar stgraph, and compute the coordinates of the vertices and bends using this numbering. 
The grid-based algorithms have two advantages: first, their performance on planar planar st-graphs has been theoretically analyzed, and second, their running times are usually low. The disadvantage is that a nonplanar DAG needs to be converted into a planar st-graph, before it can be drawn using these algorithms. This is done by introducing a fictitious vertex for each crossing between two edges. These fictitious vertices are assigned a position on the grid, but are not represented in the final drawing. The simple planarization method we have used for our study is the one described in [9]. The grid-based algorithms that we evaluated and compared fall under two categories:

Visibility Representation-Based: These algorithms use a two-step process for constructing drawings [10]. In the first step, they construct a visibility representation of the input planar st-graph. (In a visibility representation, vertices and edges are represented as horizontal and vertical linesegments, respectively; two vertices are connected by an edge if and only if they are visible to each other.) In the second step, they construct a polyline drawing of the planar st-graph from the visibility representation; this is done by replacing each vertex-segment with a point and by approximating each edge-segment with a polygonal line containing at most two bends.

The visibility representation is constructed using two numberings [12, 24]: a topological numbering of the vertices of the planar st-graph, and a topological numbering (in the dual graph) of the faces of the planar stgraph. A topological numbering of a DAG is such that for every directed edge $(u, v), v$ is assigned a higher number than $u$.

We have evaluated the performance of an algorithm, called Visibility, which follows this approach, and of three variations of it, called Barycentric Visibility, Long Edge Visibility, and Median Visibility. For our study we have used the implementations of these algorithms available in GDW [3]. The differences between Algorithms Visibility, Barycentric Visibility, Long Edge Visibility, and Median Visibility are in the strategy they use for substituting the vertex- and edge-segments of the intermediate visibility representation with the points and polygonal chains of the final drawing; they put the vertex in the middle point of the vertex-segment, in the barycenter of the endpoints of the incident edgesegments, on the endpoint of the longest incident edge-segment, and on the median of the endpoints of the incident edge-segments, respectively.

Poset-Based: These algorithms view planar st-graphs as covering graphs of partially ordered sets (posets). They exploit the relationship between the upward planarity of DAGs and the order-theoretic properties of planar lattices (see, e.g., $[16,22]$ ).

In our study we have evaluated the performance of one poset-based algorithm: the dominance drawing algorithm of [11]. This algorithm computes two topological numberings of the vertices of the input planar st- 
graph; one numbering gives the $x$-coordinates of the vertices and bends, and the other gives the $y$-coordinates. These numberings are obtained by scanning the outgoing edges of each vertex of the planar st-graph in the left-to-right and the right-to-left order respectively. For this reason, this algorithm is also known as the left-right algorithm. In this paper we have referred to this algorithm as Lattice. For our study we have used the implementation of Lattice available in $G D W[3]$.

\section{Experimental Setting}

The following quality measures of a drawing of a DAG have been considered:

Area: area of the smallest rectangle with horizontal and vertical sides covering the drawing;

Cross: total number of edge-crossings;

TotalBends: total number of edge-bends;

TotalEdgeLen: total edge length;

MaxEdgeBends: maximum number of bends on any edge;

MaxEdgeLen: maximum length of any edge;

UnifBends: standard deviation of the number of edge-bends;

UnifLen: standard deviation of the edge length;

ScreenRatio: deviation from the optimal aspect ratio, computed as the difference between the width/height ratio of the best of the two possible orientations (portrait and landscape) of the drawing and the standard 4/3 ratio of a computer screen.

ResFactor: Inverse of the minimum distance between two vertices, or two edgecrossings, or an edge-crossing and a vertex.

It is widely accepted (see, e.g., [5]) that small values of the above measures are related to the perceived aesthetic appeal and visual effectiveness of the drawing.

The issue of resolution of a drawing has been extensively studied, motivated by the finite resolution of physical rendering devices. Several papers have been published about the resolution and the area of drawings of graphs; (see, e.g., $[1,4,11,15])$. The resolution of a drawing is defined as the minimum distance between two vertices. The grid-based algorithms consider edge-bends and edge-crossings as "dummy" vertices for computing the resolution. The layeringbased algorithms, however, do not consider the edge-crossings as dummy vertices for computing the resolution. Since the measures Area, TotalEdgeLen and MaxEdgeLen of a drawing depend on its resolution, two drawings can be compared for these measures only if they have the same resolution. ResFactor allows us to scale a drawing $D_{1}$ produced by a layering-based algorithm so that it has the same resolution as that of a drawing $D_{2}$ produced by a grid-based algorithm; the scaling factor is equal to $R_{1} / R_{2}$, where $R_{1}$ and $R_{2}$ are the value of ResFactor for $D_{1}$ and $D_{2}$ respectively.

The experimental study was performed on two different sets of DAGs, both with a strong connection to "real-life" applications. We considered two typical 
contexts where DAGs play a fundamental role, namely software engineering and project planning.

The first set of test DAGs are what we call the North DAGs. They are obtained from a collection of directed graphs [21], that North collected at AT\&T Bell Labs by running for two years Draw $D A G$, an e-mail graph drawing service that accepts directed graphs formatted as e-mail messages and returns messages with the corresponding drawings [20].

Originally, the North DAGs consisted of 5114 directed graphs, whose number of vertices varied in the range $1 \ldots 7602$. However, the density of the directed graphs with a number of vertices that did not fall in the range $10 \ldots 100$ was very low (see also the statistics in [21]); since such directed graphs represent a very sparse statistical population we decided to discard them. Then we noted that many directed graphs were isomorphic; since the vertices of the directed graphs have labels associated with them, the problem is tractable. For each isomorphism class, we kept only one representative directed graph. Also, we deleted the directed graphs where subgraphs were specified as clusters, to be drawn in their own distinct rectangular region of the layout, because constrained algorithms are beyond the scope of this study. This filtering left us with 1277 directed graphs.

Still, 491 directed graphs were not connected and this was a probiem for running algorithms implemented in $G D W$ (they assume input directed graphs to be connected). Instead of discarding the directed graphs, we followed a more practical approach, by randomly adding a minimum set of directed edges that makes each directed graph connected. Finally, we made the directed graph acyclic, where necessary, by applying some heuristics for inverting the direction of a small subset of edges.

We then ran a first set of experiments and produced the statistics by grouping the DAGs by number of vertices. Although the comparison among the algorithms looked consistent (the produced plots never oddly overlapped), each single plot was not satisfactory, because it showed peaks and valleys. We went back to study the test suite and observed that grouping them by number of vertices was not the best approach. In fact, the North DAGs come from very heterogeneous sources, mainly representing different phases of various software engineering projects; as a result, directed graphs with more or less the same number of vertices may be either very dense or very sparse.

Since most of the analyzed quality measures strongly depend on the number of edges of the DAG (e.g. area, number of bends, and number of crossings), we decided that a better approach was to group the DAGs by number of edges. After some tests, we clustered the DAGs into nine groups, each with at least 40 DAGs, so that the number of edges in the DAGs belonging to group $i, 1 \leq i \leq 9$, is in the range $10 i \ldots 10 i+9$ (see Fig. 3). The resulting test suite consists of 1158 DAGs, each with edges in the range $10 \ldots 99$.

The second set of test DAGs are what we call the Pert DAGs. Although such DAGs have been randomly generated by one of the facilities of $G D W$, their construction is based on refinement operations typical of project planning. 
First, we generated a set of skeleton planar DAGs consisting of a small number of vertices to simulate the initial models of the projects. This was done by randomizing an ear-composition for each DAG. Second, we performed a random sequence of typical planning-refinement steps, i.e., expanding an edge into a series and/or a parallel component and inserting new edges between existing vertices. The inserted edges represent precedences between activities that were not captured by the starting skeleton projects.

The resulting test suite contains $813 \mathrm{DAGs}$ with edges in the range $10 \ldots 150$ and vertices in the range $10 \ldots 100$. As for the North DAGs, we grouped the Pert DAGs by number of edges, so that the number of edges in the DAGs belonging to group $i, 1 \leq i \leq 14$, is in the range $10 i \ldots 10 i+9$ (see Fig. 3).

The Pert DAGs are generally denser than the North DAGs and they are single-source single-sink. As shown in the next section, there are some quality measures for which the relative performance of the algorithms is different for the North DAGs and the Pert DAGs. Also, the plots obtained for the Pert DAGs are in general smoother, reflecting the relative uniformity of the statistical population.

\section{Analysis of the Experimental Results}

Algorithms Dot, Layers, Visibility (with its variations Barycentric Visibility, Long Edge Visibility, Median Visibility), and Lattice were executed on every North DAG and every Pert DAG, and the data for all ten quality measures were collected. Because of the different nature of the two test suites, we compared the performance of the algorithms for the North DAGs and the Pert DAGs separately. In addition, since the quality measures Area, TotalEdgeLen, and MaxEdgeLen depend upon the resolution of the drawings, we compared the layering-based and grid based algorithms separately for these three quality measures. The other seven quality measures do not depend upon the resolution, so we compared all four algorithms together for them. This gave us a total of 28 comparison charts. Figures 1- 4 display the comparison charts showing the average values for the quality measures Area, ResFactor, Cross, TotalBends, and ScreenRatio; the left column of these figures contains the charts for the North DAGs, and the right column contains the ones for the Pert DAGs. The $x$-axis of each chart shows the number of edges. The average is computed over each group of DAGs with number of edges in the range $10 \ldots 19,20 \ldots 29$, etc.

We started the experimental analysis by comparing the behavior of Visibility and its variations Barycentric Visibility, Long Edge Visibility, and Median Visibility. As a result, we found that the behavior of the visibility representationbased algorithms is almost identical for all the quality measures, with Algorithm Visibility performing slightly better than the others. In order to improve the readability of the other charts and to simplify the presentation of the experimental results, we have used Algorithm Visibility as the representative visibility representation-based algorithm. 

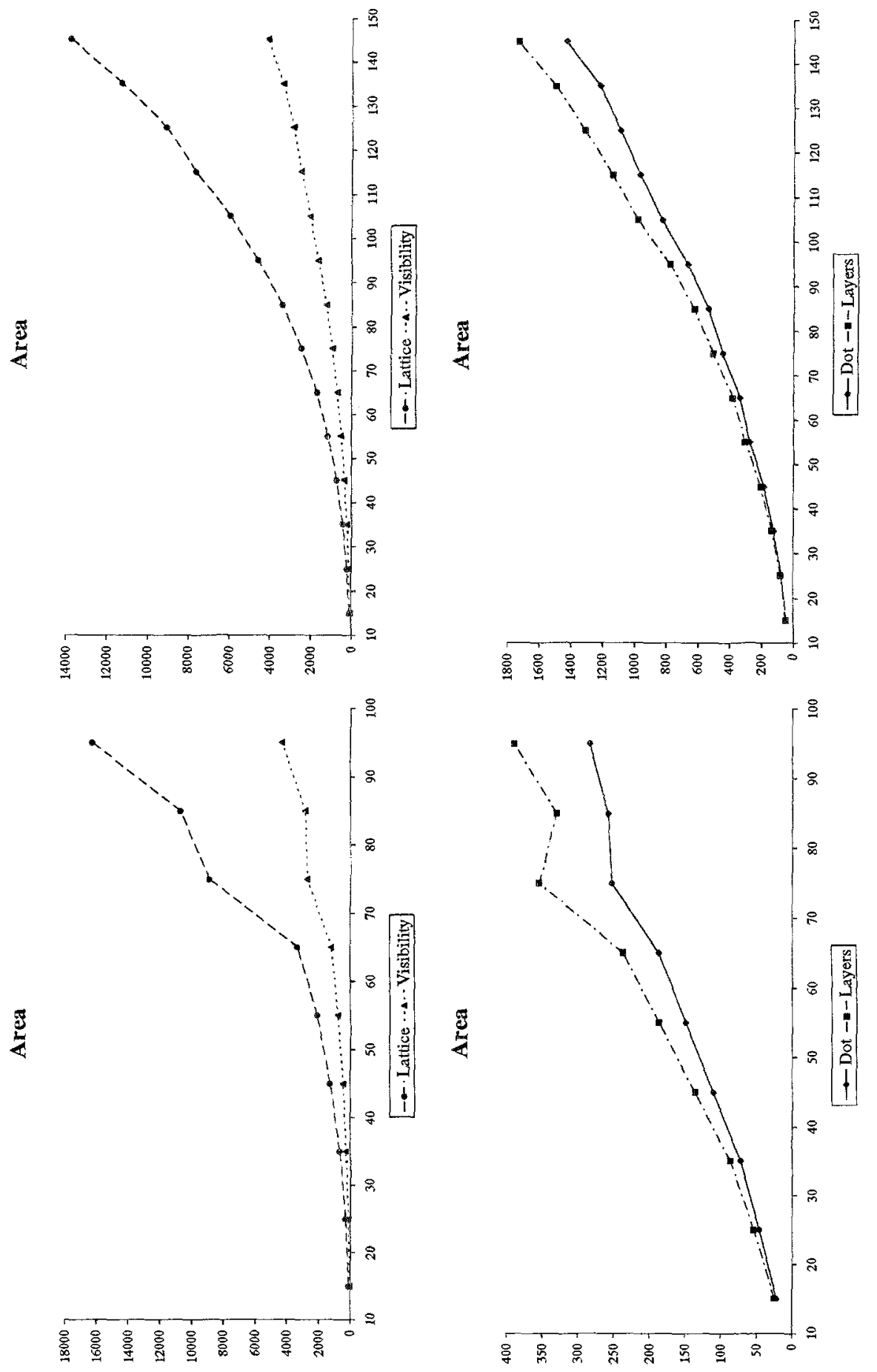

Fig. 1. Comparison charts: the $x$-axis shows the number of edges. 

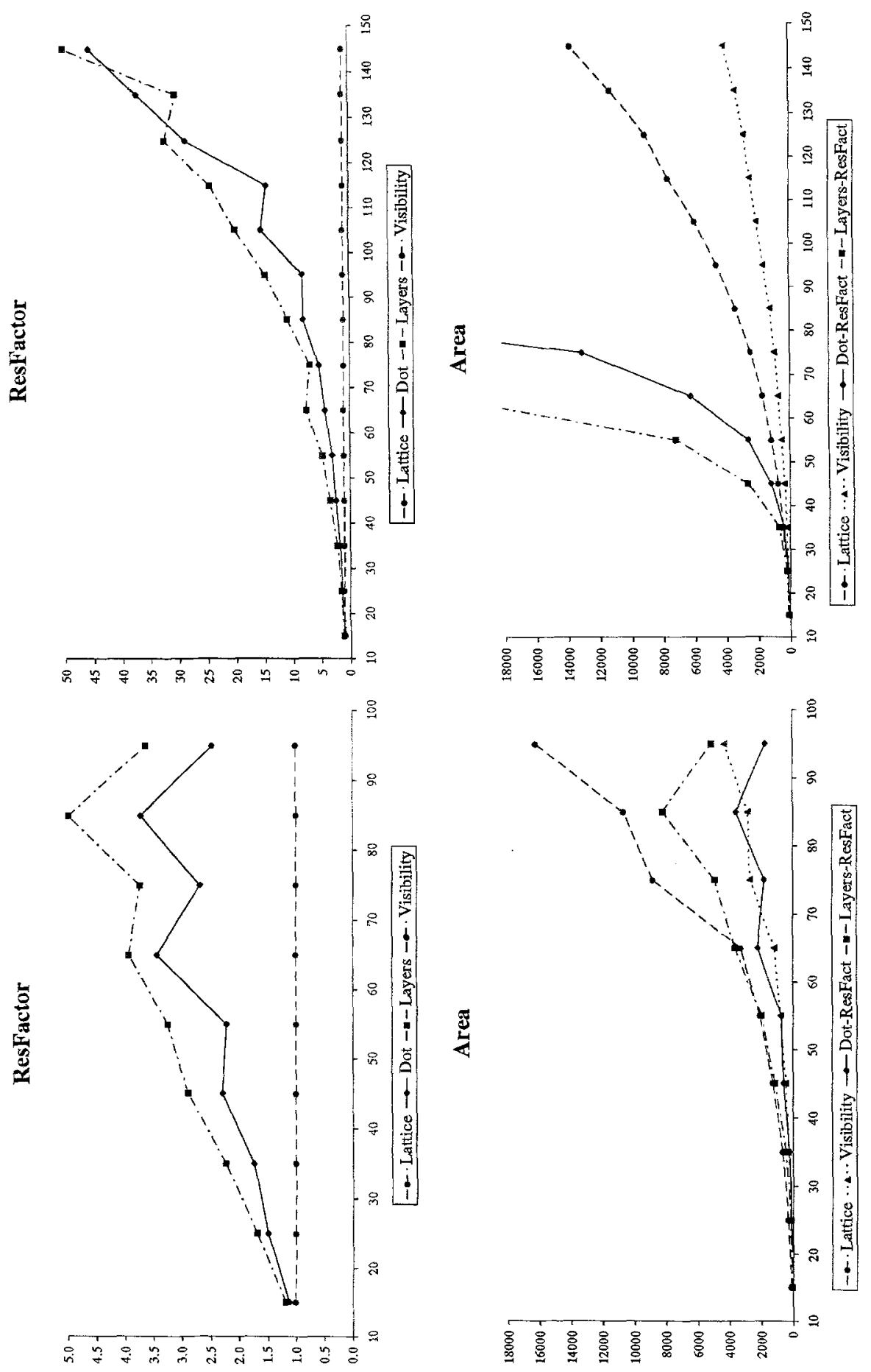

Fig. 2. Comparison charts: the $x$-axis shows the number of edges. 

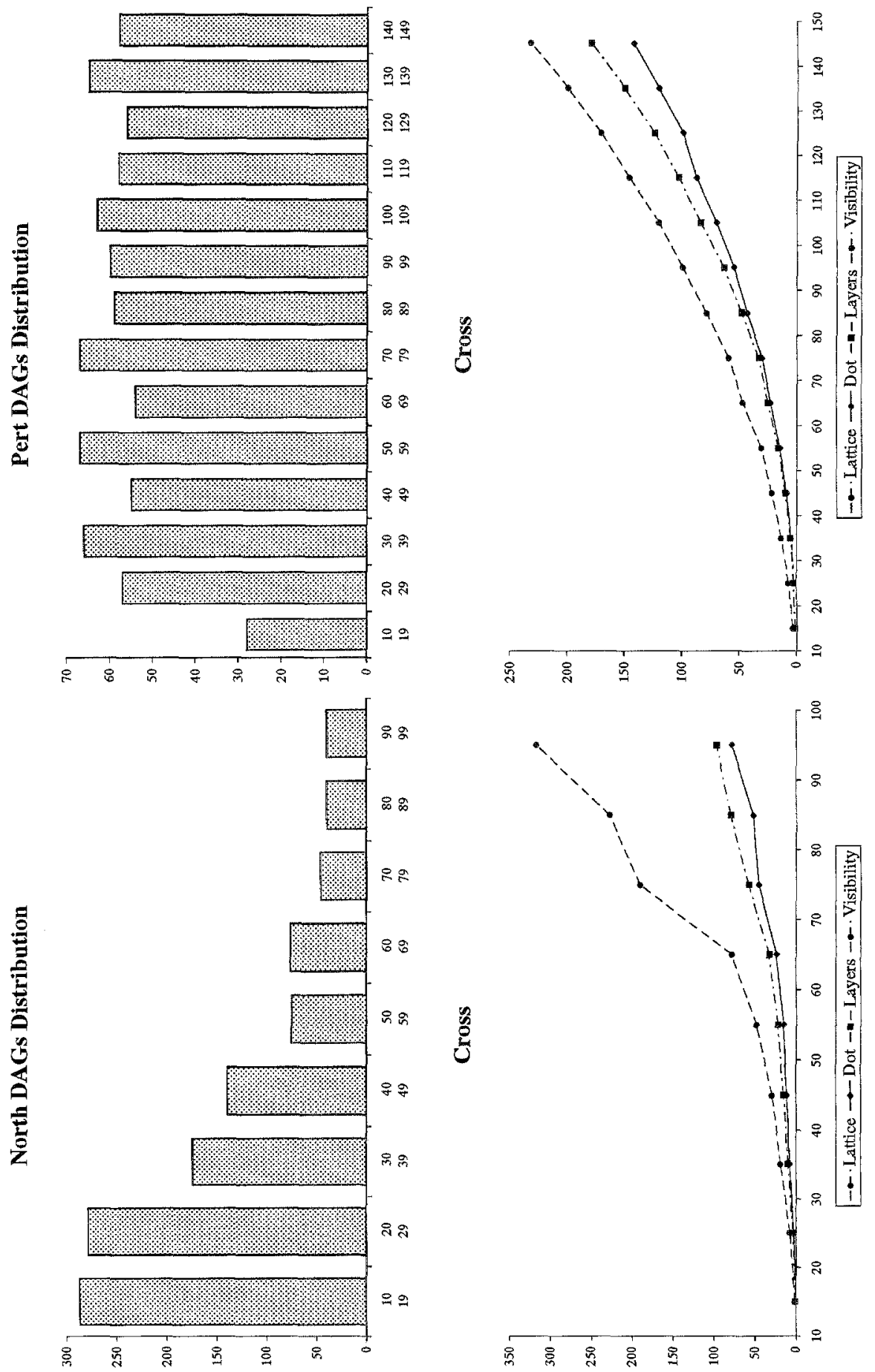

Fig. 3. Comparison charts: the $x$-axis shows the number of edges. 

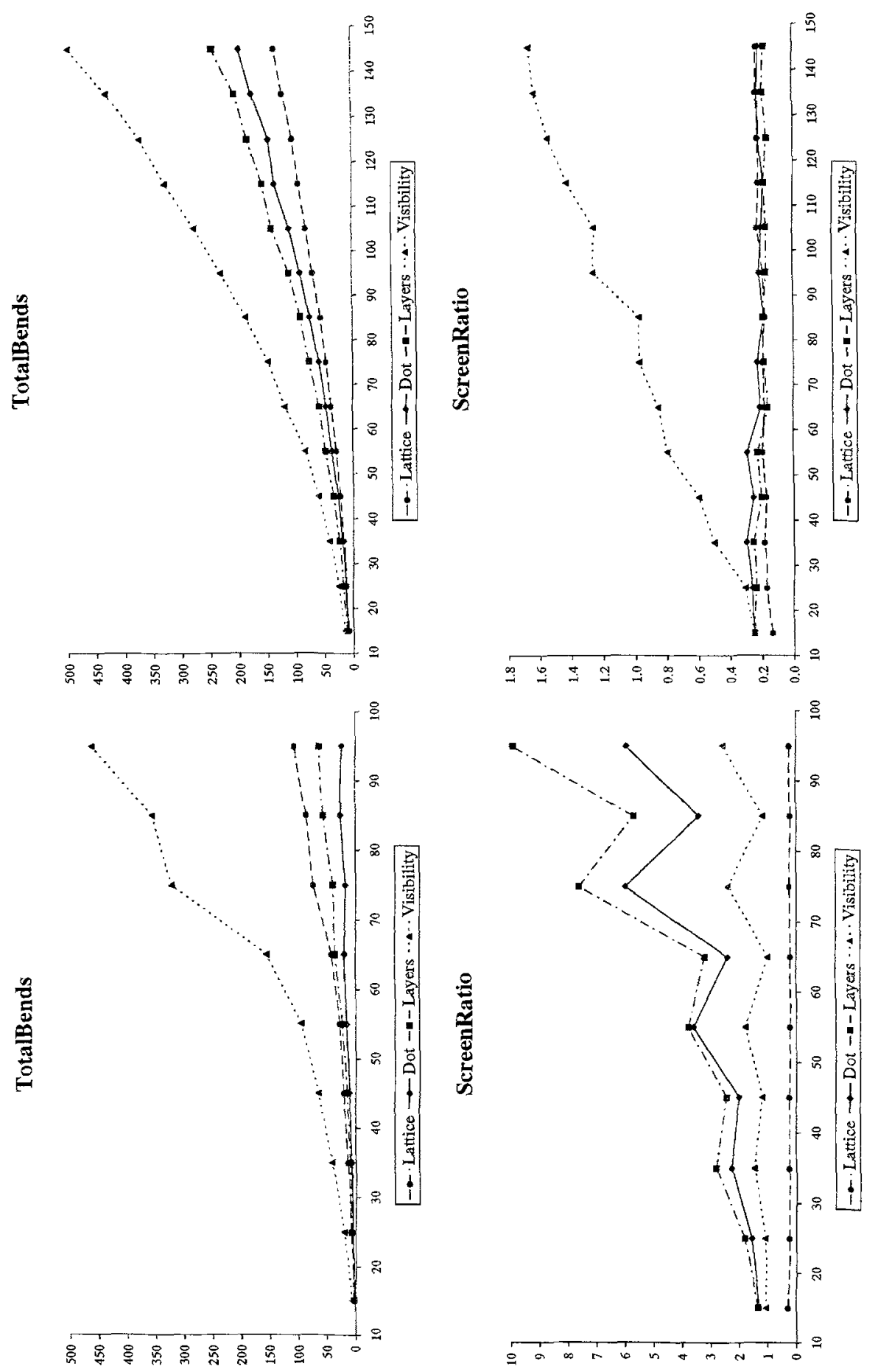

Fig. 4. Comparison charts: the $x$-axis shows the number of edges. 
The analysis of the performance of the four algorithms for each quality measure, and for each set of input DAGs is summarized below:

Area: (see Fig. 1) Dot performs better than Layers, and Lattice performs better than Visibility for both the North DAGs and the Pert DAGs. While for the North DAGs, the plots grow linearly for \#edges in the range $10 \ldots 60$, for the Pert DAGs they show quadratic growth in the entire range. Also observe that the difference in the performance of the two grid-based algorithms is significant for DAGs with more than 75 edges, whereas the two layeringbased algorithms perform about the same in the entire range.

ResFactor: (see Fig. 2) Not surprisingly, ResFactor is equal to one for the gridbased algorithms in the entire range. On the other hand, the layering-based algorithms tend to have a non-constant ResFactor. This reflects the fact that they do not take edge-crossings into consideration for defining the resolution. The bottom charts of Fig. 2 show a comparative study of the area of the drawings produced by the four algorithms. Unlike the bottom charts of Fig. 1, the plots for the two layering-based algorithms now take ResFactor into account. Note that they are comparable with the plots of the grid-based algorithms.

Cross: (see Fig. 3) Since Lattice and Visibility use the same planarizer, the drawings produced by them have the same number of edge-crossings. All the algorithms have quadratic behavior for both sets of DAGs. Dot has the best performance among the four. The difference between the performance of the layering-based and the grid-based algorithms reduces considerably for the Pert DAGs. Also observe that the slope of the plots is steeper for the Pert DAGs. This reflects the fact that the Pert DAGs are in general denser than the North DAGs and that the number of edge-crossings tend to increase with the ratio \#edges/\#vertices.

TotalBends: (see Fig. 4) The performance of Visibility is unsatisfactory. For the North DAGs, the plots of the other three algorithms grow almost linearly for \#edges up to 65 . After that, Dot is clearly the best. The experimentation with the Pert DAGs produced a surprising result. Namely, Lattice outperforms the layering-based algorithms while Visibility has still the worst behavior. As for measure Cross, the slope of the plots is steeper for the Pert DAGs. Note, however, that the behavior of Lattice seems to be quite independent from the density of the input DAG, at least for DAGs with up to 75 edges.

ScreenRatio: (see Fig. 4) Lattice seems to be the algorithm of choice with respect to this quality measure. All the algorithms have a better performance for the Pert DAGs. We believe that this a consequence of the relative density of the Pert DAGs; the drawings tend to spread in both the $x$ - and $y$-dimension.

TotalEdgeLen and MaxEdgeLen: These two measures are dependent on ResFactor. Therefore, we compared the performance of the layering-based and gridbased algorithms separately. Dot performs better than Layers, and Visibility performs better than Lattice for both the North DAGs and the Pert DAGs.

MaxEdgeBends: Quite interestingly, the plots grow linearly for the Pert DAGs 
for all four algorithms. While Dot has the best performance for the North DAGs, Lattice is the best for the Pert DAGs. The overall performance of the algorithms is much better for the North DAGs than for the Pert DAGs. Again, we believe that this is because the North DAGs are in general sparser.

\section{Cross-Fertilization of Grid- and Layering-Based Algorithms}

The analysis of the experimental results of Dot, Layers, Visibility, and Lattice clearly shows that the layering-based algorithms (Dot and Layers) produce drawings with fewer crossings than the grid-based algorithms (Visibility and Lattice). This indicates that the crossing reduction step of the layering-based algorithms is more effective than the simple planarization strategy [9] used in Visibility and Lattice. On the other hand, Visibility and Lattice perform well with respect to other quality measures (see Section 4).

The above considerations suggest the development of a hybrid strategy that substitutes the original planarization step of Visibility and Lattice with the crossing reduction step of Layers (we choose Layers over Dot for simplicity of implementation). More specifically, we first execute the crossing reduction step of Layers and then visit the resulting drawing, replacing each crossing with a fictitious vertex. This planarizes the input graph. Finally, we execute the remaining algorithmic steps of Visibility and Lattice. The new drawing algorithms so obtained will be called VisibilityLayers and LatticeLayers, respectively.

Algorithms VisibilityLayers and LatticeLayers always perform better than their "parent algorithms" Visibility and Lattice, respectively. In particular, we observe the following:

Area: The improvement of VisibilityLayers and LatticeLayers over Visibility and Lattice is especially significant for the North DAGs, where it ranges between $30 \%$ and $50 \%$.

TotalBends: Again, the improvement is especially significant (about $50 \%$ ) for the North DAGs. Also, while Layers is always better than Lattice, we have that LatticeLayers is slightly better than Layers for the North DAGs with more than 70 edges.

MaxEdgeBends: Analogous considerations to those for TotaiBends apply. Also, the improvement of VisibilityLayers over Visibility is substantial.

The analysis of the other quality measures shows similar trends.

We conclude this section by observing that the performance of the gridbased algorithms (Visibility and Lattice) is strongly influenced by the number of crossings introduced in the planarization step.

\section{Open Problems}

Our experiments lead to many interesting theoretical and practical questions: 
- The angular resolution of a drawing is the magnitude of the smallest angle between any two edges incident on a vertex. The readability of a drawing can be improved by increasing its angular resolution. Unfortunately, not much is known either theoretically or empirically about the angular resolution of drawings of directed graphs. This issue is worth exploring.

- Dot in a final step converts the polylines into Bezier curves using splines. This has a dramatic impact on the quality of the drawing. Similarly, we believe that the performance of several algorithms, such as Visibility, can be improved by a postprocessing "beautification" step. For example, it would be interesting to study bend-stretching techniques [25] that reduce the bends by doing local transformations.

- Similarly, the role of the preprocessing step should also be studied. In particular, the performance of grid-based algorithms can be improved by using a more sophisticated planarizer.

\section{Acknowledgements}

We are grateful to Stephen North for many discussions about Dot and the collection of directed graphs. We thank Petra Mutzel for answering our questions about the statistics on such graphs. Finally we thank Paola Vocca for her encouragement during the writing of this paper.

\section{References}

1. P. Bertolazzi, R. F. Cohen, G. Di Battista, R. Tamassia, and I. G. Tollis. How to draw a series-parallel digraph. Internat. J. Comput. Geom. Appl., 4:385-402, 1994.

2. F. J. Brandenburg, M. Himsolt, and C. Rohrer. An experimental comparison of force-directed and randomized graph drawing algorithms. In F. J. Brandenburg, editor, Graph Drawing (Proc. GD '95), volume 1027 of Lecture Notes in Computer Science, pages 76-87. Springer-Verlag, 1996.

3. L. Buti, G. Di Battista, G. Liotta, E. Tassinari, F. Vargiu, and L. Vismara. GDWorkbench: A system for prototyping and testing graph drawing algorithms. In F. J. Brandenburg, editor, Graph Drawing (Proc. GD '95), volume 1027 of LNCS, pages 111-122. Springer-Verlag, 1996.

4. M. Chrobak, M. T. Goodrich, and R. Tamassia. Convex drawings of graphs in two and three dimensions. In Proc. 12th Annw. ACM Sympos. Comput. Geom., pages 319-328, 1996.

5. G. Di Battista, P. Eades, R. Tamassia, and I. G. Tollis. Algorithms for drawing graphs: An annotated bibliography. Comput. Geom. Theory Appl., 4:235-282, 1994.

6. G. Di Battista, A. Garg, G. Liotta, A. Parise, R. Tamassia, E. Tassinari, F. Vargiu, and L. Vismara. Drawing directed graphs: An experimental study. Technical Report CS-96-24, Center for Geometric Computing, Dept. Computer Science, Brown Univ., 1996. ftp://ftp.es.bromn.edu/pub/techreports/96/ cs96-24.ps.z. 
7. G. Di Battista, A. Garg, G. Liotta, R. Tamassia, E. Tassinari, and F. Vargiu. An experimental comparison of three graph drawing algorithms. In Proc. 11th Annu. ACM Sympos. Comput. Geom., pages 306-315, 1995.

8. G. Di Battista, A. Garg, G. Liotta, R. Tamassia, E. Tassinari, and F. Vargiu. An experimental comparison of four graph drawing algorithms. Comput. Geom. Theory Appl., 1996 (to appear). http:// ॠни.cs.bronn.edu/cgc/papers/ dglttr-ecfgd-96.ps.gz.

9. G. Di Battista, E. Pietrosanti, R. Tamassia, and I. G. Tollis. Automatic layout of PERT diagrams with XPERT. In Proc. IEEE Workshop on Visual Languages (VL'89), pages 171-176, 1989.

10. G. Di Battista and R. Tamassia. Algorithms for plane representations of acyclic digraphs. Theoret. Comput. Sci., 61:175-198, 1988.

11. G. Di Battista, R. Tamassia, and I. G. Tollis. Area requirement and symmetry display of planar upward drawings. Discrete Comput. Geom, 7:381-401, 1992.

12. G. Di Battista, R. Tamassia, and I. G. Tollis. Constrained visibility representations of graphs. Inform. Process. Lett., 41:1-7, 1992.

13. E. R. Gansner, E. Koutsofios, S. C. North, and K. P. Vo. A technique for drawing directed graphs. IEEE Trans. Softw. Eng., 19:214-230, 1993.

14. E. R. Gansner, S. C. North, and K. P. Vo. DAG - A program that draws directed graphs. Softw. - Pract. Exp., 18(11):1047-1062, 1988.

15. A. Garg and R. Tamassia. Planar drawings and angular resolution: Algorithms and bounds. In J. van Leeuwen, editor, Algorithms (Proc. ESA '94), volume 855 of Lecture Notes in Computer Science, pages 12-23. Springer-Verlag, 1994.

16. A. Garg and R. Tamassia. Upward planarity testing. Order, 12:109-133, 1995.

17. M. Himsolt. Comparing and evaluating layout algorithms within GraphEd. $J$. Visual Lang. Comput., 6(3), 1995. (Special Issue on Graph Visualization, I. F. Cruz and P. Eades, editors).

18. S. Jones, P. Eades, A. Moran, N. Ward, G. Delott, and R. Tamassia. A note on planar graph drawing algorithms. Technical Report 216, Department of Computer Science, University of Queensland, 1991.

19. M. Jünger and P. Mutzel. Exact and heuristic algorithms for 2-layer straightline crossing minimization. In F. J. Brandenburg, editor, Graph Drawing (Proc. GD '95), volume 1027 of Lecture Notes in Computer Science, pages 337-348. SpringerVerlag, 1996.

20. E. Koutsofios and S. North. Drawing graphs with dot, 1993. dot user's manual. ftp://ftp.research. att. com/dist/drandag/.

21. S. North. 5114 directed graphs, 1995. Manuscript. ftp://ftp.research.att.com/ dist/drandag/.

22. I. Rival. Reading, drawing, and order. In I. G. Rosenberg and G. Sabidussi, editors, Algebras and Orders, pages 359-404. Kluwer Academic Publishers, 1993.

23. K. Sugiyama, S. Tagawa, and M. Toda. Methods for visual understanding of hierarchical systems. IEEE Trans. Syst. Man Cybern., SMC-11(2):109-125, 1981.

24. R. Tamassia and I. G. Tollis. A unified approach to visibility representations of planar graphs. Discrete Comput. Geom., 1(4):321-341, 1986.

25. R. Tamassia and I. G. Tollis. Planar grid embedding in linear time. IEEE Trans. Circuits Syst., CAS-36(9):1230-1234, 1989. 